\title{
BMJ Open Medicines prescribed by non-medical independent prescribers in primary care in Wales: a 10-year longitudinal study April 2011-March 2021
}

\author{
Paul Deslandes (10 , ${ }^{1,2}$ Hannah Blowers, ${ }^{3}$ Kath Haines, ${ }^{1}$ Karen Hodson, ${ }^{3}$ \\ Rhian Deslandes ${ }^{3}$
}

To cite: Deslandes $\mathrm{P}$, Blowers $\mathrm{H}$, Haines $\mathrm{K}$, et al. Medicines prescribed by non-medical independent prescribers in primary care in Wales: a 10year longitudinal study April 2011-March 2021. BMJ Open 2022;12:e059204. doi:10.1136/ bmjopen-2021-059204

- Prepublication history for this paper is available online. To view these files, please visit the journal online (http://dx.doi. org/10.1136/bmjopen-2021 059204).

Received 16 November 2021 Accepted 02 February 2022

Check for updates

(C) Author(s) (or their employer(s)) 2022. Re-use permitted under CC BY-NC. No commercial re-use. See rights and permissions. Published by BMJ.

${ }^{1}$ All Wales Therapeutics and Toxicology Centre, Academic Centre, University Hospital Llandough, Llandough, UK ${ }^{2}$ School of Care Sciences, University of South Wales, Treforest, UK

${ }^{3}$ Cardiff School of Pharmacy and Pharmaceutical Sciences, Cardiff University, Cardiff, UK

Correspondence to

Dr Rhian Deslandes:

deslandesre@cardiff.ac.uk

\section{ABSTRACT}

Objectives The therapeutic classes of medicines prescribed by non-medical independent prescribers (NMIPs) working in primary care in Wales has not been studied in detail. The aim of this study was to conduct a 10-year longitudinal analysis of NMIP prescribing in Wales from April 2011 to March 2021. The study examined the British National Formulary (BNF) chapters from which medicines were prescribed by NMIPS, whether this changed over time, and whether there was variation in prescribing across the geographic regions of Wales. Design Retrospective secondary data analysis of primary care prescribing data. Monthly prescribing data for the 10 National Health Service financial years (April to March) from April 2011 to March 2021 were obtained from the Comparative Analysis System for Prescribing Audit software. Data were analysed according to BNF chapter, to identify in which therapeutic areas NMIPs were prescribing, and whether this changed over the study period.

Results The number of items prescribed by NMIPs increased during the study period. From April 2011 to March 2021 prescribing in seven BNF chapters equated to approximately $80 \%$ of total items, with cardiovascular system medicines most prescribed. In the financial year 2011-2012 the BNF chapters with the greatest proportion of items prescribed were infection (18\%) and respiratory system (13\%), while in 2020-2021, these had changed to cardiovascular $(23 \%)$ and nervous system (19\%). The number of items prescribed in each health board in Wales varied, however, the BNF chapters contributing the largest percentages of items to the health board totals were broadly comparable.

Conclusions The BNF chapter with the most prescribed items changed from infection to cardiovascular during the study period, suggesting an increase in chronic disease management by NMIPs. The impact of this on the delivery of primary care services and patient outcomes is a focus for future work.

\section{INTRODUCTION}

In the UK, prescribing practice has changed significantly since the 1980s. Legislative changes in the 1990s and early 2000s granted prescribing rights to non-medical healthcare
Strengths and limitations of this study

- This study used an All Wales database, covering 10 years of non-medical independent prescribing data across the whole population in primary care.

- The data obtained allowed trends and regional variation in prescribing to be identified.

- The secondary data used did not allow the reasons for any observed changes in prescribing to be investigated.

- Patient level data were not available, therefore, it was not possible to determine prescribing patterns for individual patients.

professionals, with appropriate experience in their relevant scope of practice who had completed an accredited course of training. ${ }^{1}$ Non-medical independent prescribing, defined as: "prescribing by a practitioner (eg, nurse, pharmacist) responsible and accountable for the assessment of patients with undiagnosed or diagnosed conditions and for decisions about the clinical management required, including prescribing.' was first introduced for pharmacists and nurses in $2006 .{ }^{2}$ The intention was to advance and develop the healthcare system, by improving patient access to medicines, and enhancing patient care and experience, without reducing safety. ${ }^{34}$ In parallel, the roles and responsibilities of non-medical healthcare professionals have evolved, to facilitate support for a more flexible multidisciplinary healthcare team. ${ }^{13}$

Following implementation of non-medical prescribing, the benefits have been recognised widely by both patients and prescribers themselves. Patients report satisfaction and positive experiences including increased flexibility and accessibility, ${ }^{56}$ and good rapport, ${ }^{7}$ while practitioners report improved autonomy and job satisfaction despite certain barriers. ${ }^{8}$ It has also been noted that non-medical 
independent prescribers (NMIPs) relieve pressures on general practitioners (GPs). ${ }^{9}$ However, various barriers to the uptake and implementation of non-medical independent prescribing still exist, such as lack of support and confidence, leading to inconsistencies both across and within organisations. ${ }^{1011}$

In Wales, primary care National Health Service (NHS) services are provided through seven local health boards (LHBs). As a way to manage increasing demand for primary care services in Wales, Welsh Government implemented 64 primary care clusters in $2015 .^{12}$ Clusters are composed of groups of adjacent general practices and partner organisations, such as community pharmacies, working together to collaboratively provide services locally. ${ }^{13}$ Implementation aimed to refocus primary care and improve patients' access to healthcare, while relieving pressure on NHS hospitals. In 2018, Welsh Government published a vision for health and social care, 'A Healthier Wales', which outlines the aim to move away from providing care in hospitals to instead providing care in the community. ${ }^{14}$ All of these initiatives were potential drivers for greater uptake of NMIPs into primary care in Wales. ${ }^{815}$

Previous analysis of prescribing trend data for Wales has shown that the number of NMIPs prescribing in primary care increased from 2011 to 2018. The total volume of items prescribed as well as the volume prescribed by NMIPs grew over the same period, with a significant increase in the prescribing rate for NMIPs following the implementation of primary care clusters. ${ }^{15}$ While the study of Alghamdi et al explored the broad prescribing trend, it did not identify the specific groups of medicines prescribed, and by implication, the therapeutic areas in which NMIPs were working. The aim of this study was to conduct a 10-year longitudinal analysis of NMIP prescribing in Wales from April 2011 to March 2021. Specifically, the study examined the British National Formulary (BNF) chapters from which medicines were prescribed by NMIPs in primary care in Wales, whether this changed over time, and whether there was variation in prescribing across the different geographical regions of Wales.

\section{METHODS}

\section{Data source}

This was a retrospective secondary data analysis of monthly prescribing data issued by NMIPs in primary care in Wales. Prescribing data were accessed from the Comparative Analysis System for Prescribing Audit (CASPA) software. CASPA software records all WP10 prescriptions (Welsh NHS prescriptions) dispensed in community pharmacies and submitted to NHS Wales Prescribing Services for financial reimbursement. Although the term 'prescribing' is used throughout this paper, the data represents prescriptions that have been issued, dispensed and forwarded for pricing. ${ }^{16}$ As a result, dispensing may not reflect prescribing as some patients may not get their prescriptions dispensed. However, the abolition of the prescription charge in Wales in 2007 helped to reduce the percentage of patients who failed to have a prescription dispensed from an estimated $8.9 \%$ to an estimated $5.5 \%,{ }^{17}$ reducing the impact of this confounder.

\section{Prescribing measure}

For the purpose of this study, the number of prescription items was the chosen prescribing measure for prescribing volume. This measure was chosen, as it provides an indication of the extent of the prescriber's activity, as each medicine prescribed counts as an item, irrespective of the amount, or the cost. Welsh LHBs vary greatly by population size, ${ }^{18}$ which is reflected in variation in GP prescribing data. However, as non-medical independent prescribing was a developing service, it was felt that the amount of prescribing by NMIPs would be more dependent on the number of practising NMIPs in each LHB, which might or might not have been related to the population of the LHB. For example, the number of items prescribed in an LHB with a small population but a large number of NMIPs would likely be greater than that in an LHB with a large population but a small number of NMIPs. NMIP prescribing in each LHB for the financial year 2020-2021 was correlated with the mid-year population estimate for the LHB (using population data for that year available at https://statswales.gov.wales/Catalogue/ Population-and-Migration/Population/Estimates/LocalHealth-Boards/populationestimates-by-lhb-age) to investigate this assumption.

\section{Data collection}

Nationwide monthly prescribing data for the 10 NHS financial years (April to March) from April 2011 to March 2021 were obtained for NMIPs in Wales. It was not possible to differentiate between healthcare professions, therefore the data represents prescribing by all NMIPs in primary care rather than a specific professional group. Data were synthesised to financial years and filtered by LHB to analyse the non-medical independent prescribing trends by geographical location. During the period of study, the structure of two LHBs changed, with part of the previous Abertawe Bro Morgannwg University Health Board (now Swansea Bay University Health Board) becoming part of Cwm Taf Morgannwg University Health Board in April 2019. The data presented represents prescribing that occurred for these organisations as they were structured during each financial year. As a result, prescribing in Swansea Bay after March 2019 may be lower than might have been expected, and prescribing in Cwm Taf Morgannwg greater than might have been expected, based on previous trends.

Prescribing data for drugs available in CASPA are largely arranged in a way that corresponds to the chapters in the BNF. As a result, prescribing was divided between 15 chapters: gastrointestinal system, cardiovascular system, respiratory system, nervous system, infection, endocrine system, genitourinary system, immune system 
and malignant disease, blood and nutrition, musculoskeletal system, eye, ear, nose and throat, skin, vaccines and anaesthesia. ${ }^{19}$ However, in addition, CASPA holds details of items such as dressings, appliances and certain other preparations (eg, substances used in medicines formulation), which are categorised beyond the BNF chapter structure. In this study, these items were grouped together, and included as 'other' items. This 'other' group also included a small number of items used in the management of poisoning. BNF chapter 4 (nervous system) includes medicines for the treatment of pain, as well as those for psychological and neurological illnesses, such as depression, psychosis and Parkinson's disease. As part of the analysis of this chapter, the number of items recorded in the analgesia section was compared with the number of items in the other sections of this chapter in order to differentiate between therapeutic areas.

\section{Data analysis}

Data were presented in graphs and figures using descriptive statistics, where appropriate. Pareto analysis was used to provide a more focused exploration of the data. Pareto charts display the total number of items prescribed within each BNF chapter in descending order of quantity and an overlaid line displays the cumulative percentage of the total prescribing. A Pareto analysis is commonly used to focus investigations on the most significant categories when the data is defined by a large variety of categories, and for this reason, was chosen for this study. A Pareto analysis of all items prescribed from April 2011 to March 2021 (the whole dataset) as well as 2011-2012 (the first year of data) and 2020-2021 (the final year of data), was used to identify the chapters which encompassed $80 \%$ of the total prescribing by NMIPs. Pearson correlation analysis was used to compare prescribing volume in 20202021 and health board population (analysis performed using GraphPad Prism V.5.04, GraphPad Software, California, USA).

\section{Patient and public involvement}

This research was done without patient and public involvement. Patients were not invited to comment on the study design and were not consulted to develop patient relevant outcomes or interpret the results. Patients were not invited to contribute to the writing or editing of this document for readability or accuracy.

\section{RESULTS}

Number of items prescribed by BNF chapter in Wales

The total number of items prescribed by NMIPs and dispensed in community pharmacy from April 2011 to March 2021 (referred to as the whole study period) was approximately 11.2 million. The number of items in 2011-2012 was approximately 417000 , which increased to approximately 2.2 million in 2020-2021 (an increase of $430 \%$ ). The number of items prescribed during the whole study period varied according to BNF chapter, ranging from 2184656 items prescribed for medicines within chapter 2 (cardiovascular system) to 15995 items prescribed for medicines within chapter 15 (anaesthesia). A Pareto analysis (figure 1) identified that approximately $80 \%$ of the total number of items prescribed during the whole study period equated to seven BNF chapters:

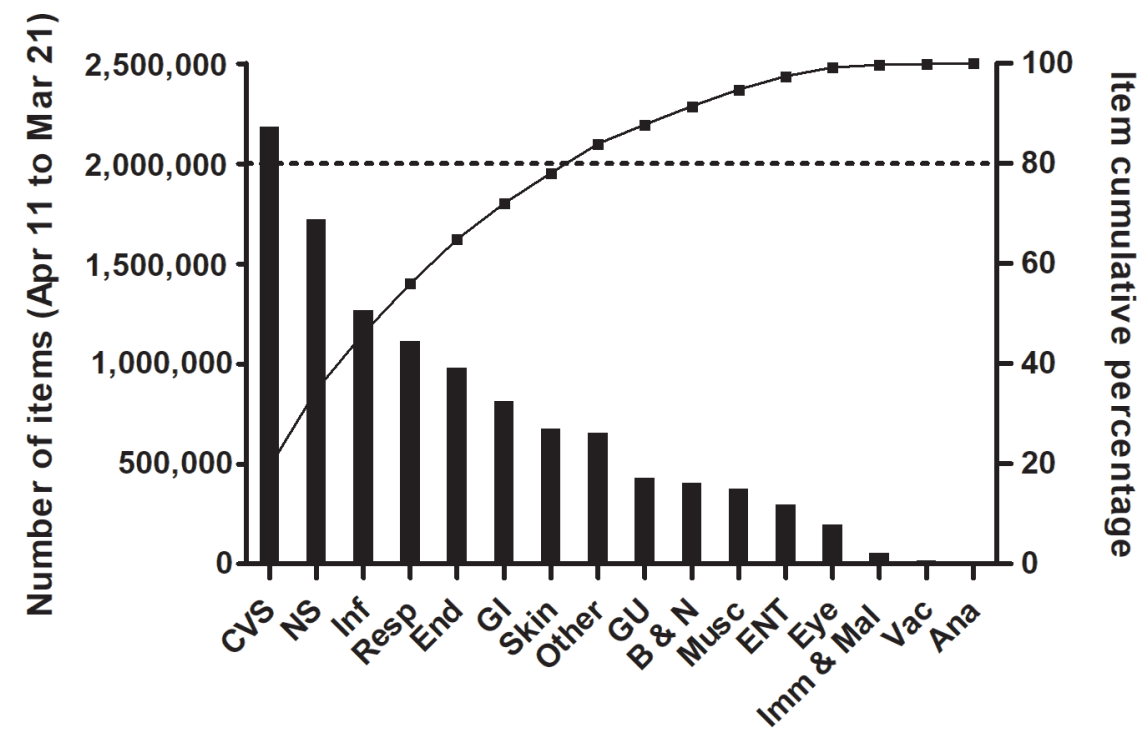

Therape utic area

Figure 1 Pareto analysis of the total number of items prescribed by an NMIP in Wales and dispensed in a community pharmacy from April 2011 to March 2021 by BNF chapter. The columns on the left axis represent items per BNF chapter. The line on the right axis shows a cumulative percentage of the number of items prescribed. The BNF chapters are abbreviated as: Ana, anaesthesia; B \& N, blood and nutrition; CVS, cardiovascular system; ENT, ears, nose and throat; End, endocrine system; GI, gastrointestinal; GU, genitourinary system; Inf, infection; Imm \& Mal, immune system and malignant disease; Musc, musculoskeletal system; NS, nervous system; Resp, respiratory system; Vac, vaccines. 


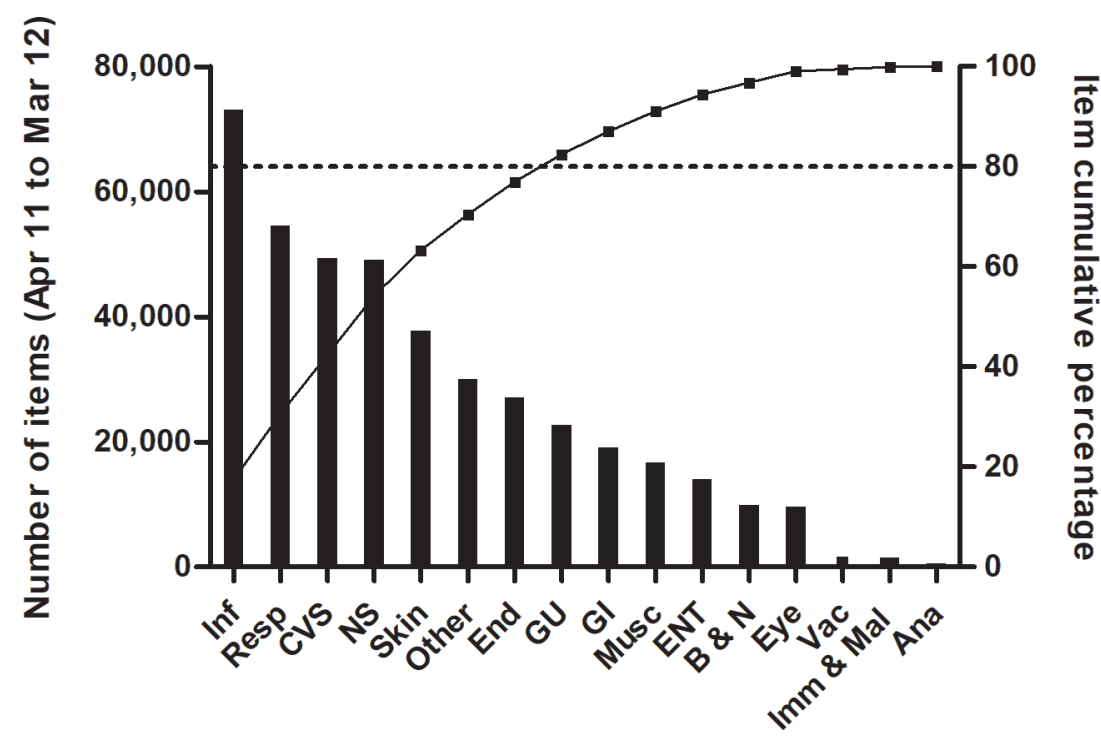

The rape utic area

Figure 2 Pareto analysis of the total number of items prescribed by an NMIP in Wales and dispensed in a community pharmacy from April 2011 to March 2012 by BNF chapter. The columns on the left axis represent items per BNF chapter. The line on the right axis shows a cumulative percentage of the number of items prescribed. The BNF chapters are abbreviated as: Ana, anaesthesia; B \& N, blood and nutrition; CVS, cardiovascular system; ENT, ears, nose and throat; End, endocrine system; Gl, gastrointestinal; GU, genitourinary system; Inf, infection; Imm \& Mal, immune system and malignant disease; Musc, musculoskeletal system; NS, nervous system; Resp, respiratory system; Vac, vaccines.

cardiovascular system, nervous system, infection, respiratory system, endocrine system, gastrointestinal system and skin. Prescribing within three of the BNF chapters, immune system and malignant disease, vaccines and anaesthesia was minimal.

Pareto analysis for prescribing data in 2011-2012, identified that eight BNF chapters accounted for approximately
$80 \%$ of the total number of items prescribed: infection, respiratory system, cardiovascular system, nervous system, skin, 'other', endocrine and genitourinary (figure 2). In 2020-2021, the seven BNF chapters that accounted for approximately $80 \%$ of prescribing were cardiovascular system, nervous system, endocrine system, gastrointestinal system, respiratory system, infection and skin (figure 3).

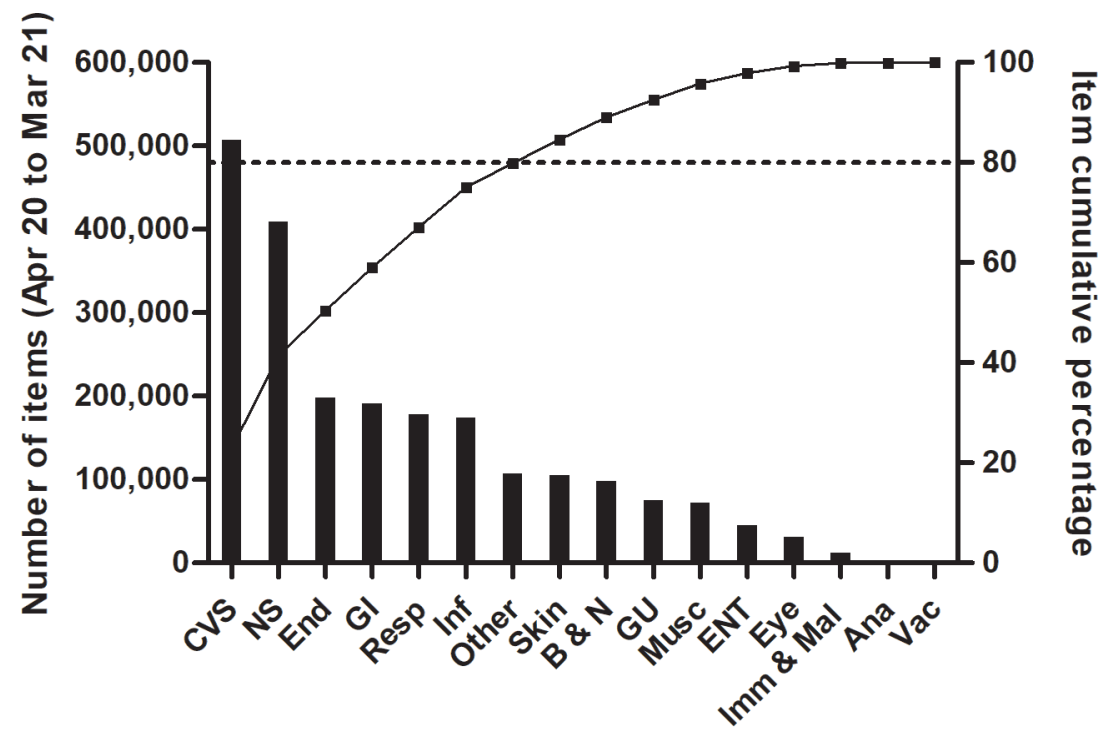

The rape utic area

Figure 3 Pareto analysis of the total number of items prescribed by an NMIP in Wales and dispensed in a community pharmacy from April 2020 to March 2021 by BNF chapter. The columns on the left axis represent items per BNF chapter. The line on the right axis shows a cumulative percentage of the number of items prescribed. The BNF Chapters are abbreviated as: Ana, anaesthesia; B \& N, blood and nutrition; CVS, cardiovascular system; ENT, ears, nose and throat; End, endocrine system; Gl, gastrointestinal; GU, genitourinary system; Inf, infection; Imm \& Mal, immune system and malignant disease; Musc, musculoskeletal system; NS, nervous system; Resp, respiratory system; Vac, vaccines. 


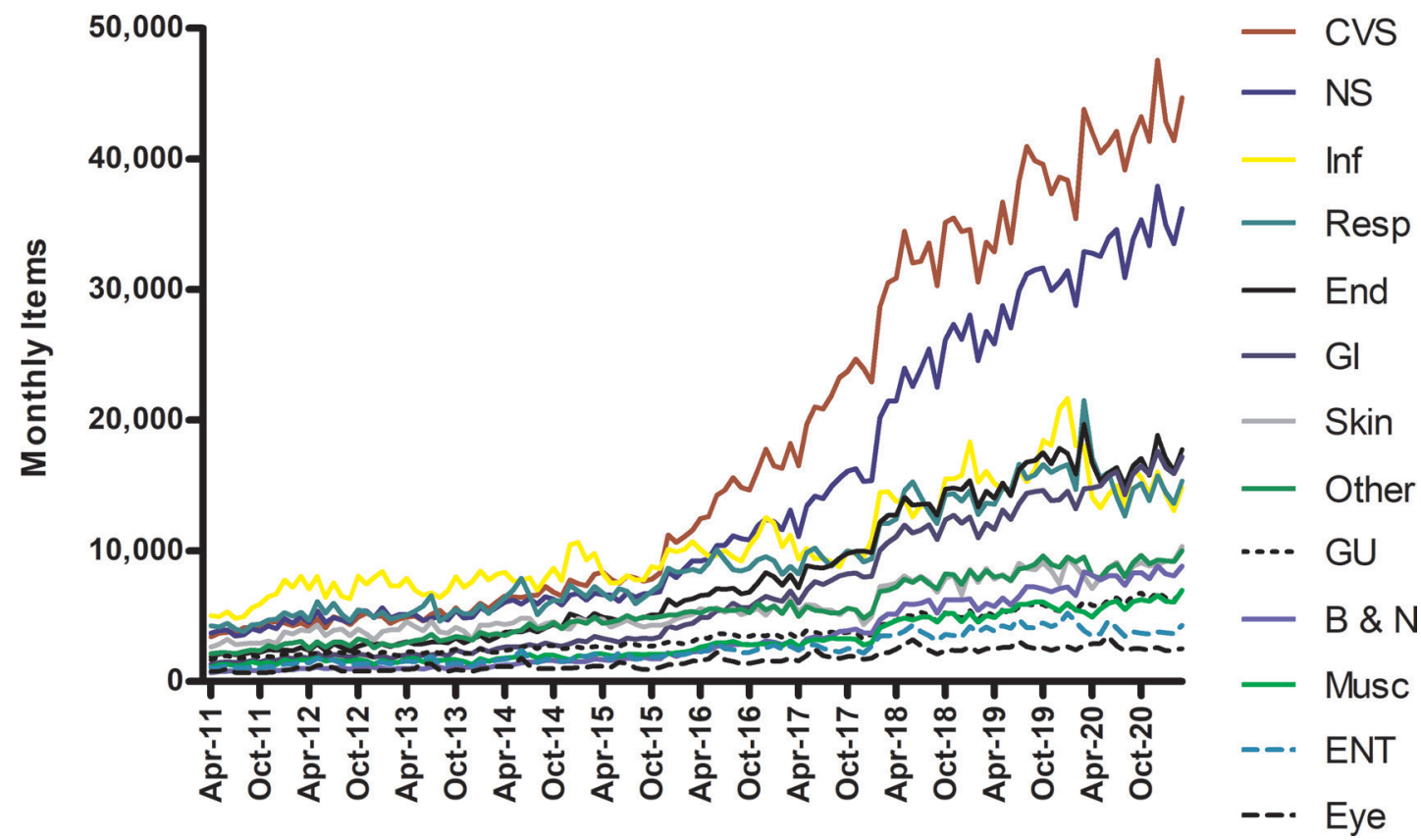

Figure 4 Trend in the total number of items prescribed by an NMIP in Wales and dispensed in a community pharmacy by BNF category. The BNF chapters are abbreviated as: The three chapters with the smallest usage (immune system and malignant disease, anaesthesia, and vaccines), have been removed to aid clarity. B \& N, blood and nutrition; CVS, cardiovascular system; ENT, ears, nose and throat; End, endocrine system; GI, gastro-intestinal; GU, genito-urinary system; Inf, infection; Musc, musculoskeletal system; NS, nervous system; Resp, respiratory system.

In 2011-2012, infection and respiratory system were the top two chapters, and accounted for approximately $18 \%$ and $13 \%$ of prescribing respectively. In 2020-2021, these chapters were ranked sixth and fifth, respectively, and each accounted for approximately $8 \%$ of all items $(16 \%$ for the two chapters combined). Prior to the COVID-19 pandemic in both 2018-2019 and 2019-2020, infection accounted for $10 \%$ of prescribed items, and was the third ranked chapter. In 2020-2021, cardiovascular system and nervous system were the top two chapters, and accounted for approximately $40 \%$ of items prescribed. This was an increase from $24 \%$ of all items in 2011-2012, where they were ranked third and fourth, respectively. Within the nervous system chapter, in 2011-2012, analgesics accounted for $50 \%$ of prescribed items, while in 2020 2021, they accounted for $34 \%$ of prescribed items.

\section{Prescribing trend over time}

The trend in prescribing in Wales according to BNF chapter is shown in figure 4, with figures 5 and 6 highlighting the prescribing trend from the middle and lower chapters, respectively. Prescribing showed a year-onyear increase from 2011-2012 to 2019-2020 in all BNF chapters, with the exception of vaccines, and a small fall $(0.3 \%)$ in infection in 2017-2018 compared with 20162017. Prescribing in five chapters (respiratory, infection, ear, nose and throat, vaccines, and anaesthesia) was lower in 2020-2021 than in 2019-2020, but with the exception of vaccines, remained higher than in 2011-2012. The chapters with the largest percentage difference in prescribing between 2011-2012 and 2020-2021 were cardiovascular (928\%), gastrointestinal (899\%) and blood and nutrition (894\%). The greatest year-on-year increase in overall prescribing $(45 \%)$ occurred between 2017-2018 and 2018-2019, while the smallest year-on-year increase (5\%) was between 2019-2020 and 2020-2021. The largest increase in cardiovascular system prescribing (70\%) occurred between 2016-2017 and 2017-2018, and nervous system prescribing (59\%) between 2017-2018 and 2018-2019.

\section{Number of items prescribed by LHB}

The LHB with the largest amount of prescribing by NMIPs throughout the whole study period, and in 2011-2012 and 2020-2021 was Betsi Cadwaladr. The lowest prescribing LHB over the whole study period and in 2011-2012 was Powys, while the lowest prescribing in 2020-2021 was in Cwm Taf Morgannwg (see table 1). Prescribing in each health board in 2020-2021 was weakly correlated with the mid-year population estimate for the health board (Pearson $\left.\mathrm{r}^{2}=0.66, \mathrm{p}<0.05\right)$. Prescribing in the financial years prior to and during the COVID-19 pandemic is also shown in table 1 . For Wales, the percentage difference between 2011 and 2012 was 404\%, and from 20112012 to $2020-2021$ was $430 \%$. The largest reduction in prescribing between 2019-2020 and 2020-2021 was seen in Hywel Dda $(-35 \%)$, while the largest increase was seen in Cardiff and Vale $(45 \%)$.

Within each LHB, the BNF chapters contributing the largest percentage of items to the total prescribing are shown in table 2. In 2011-2012, infection contributed the largest percentage to the total prescribing in five of the seven LHBs, and respiratory system and nervous system contributed the largest percentage in Cardiff and 


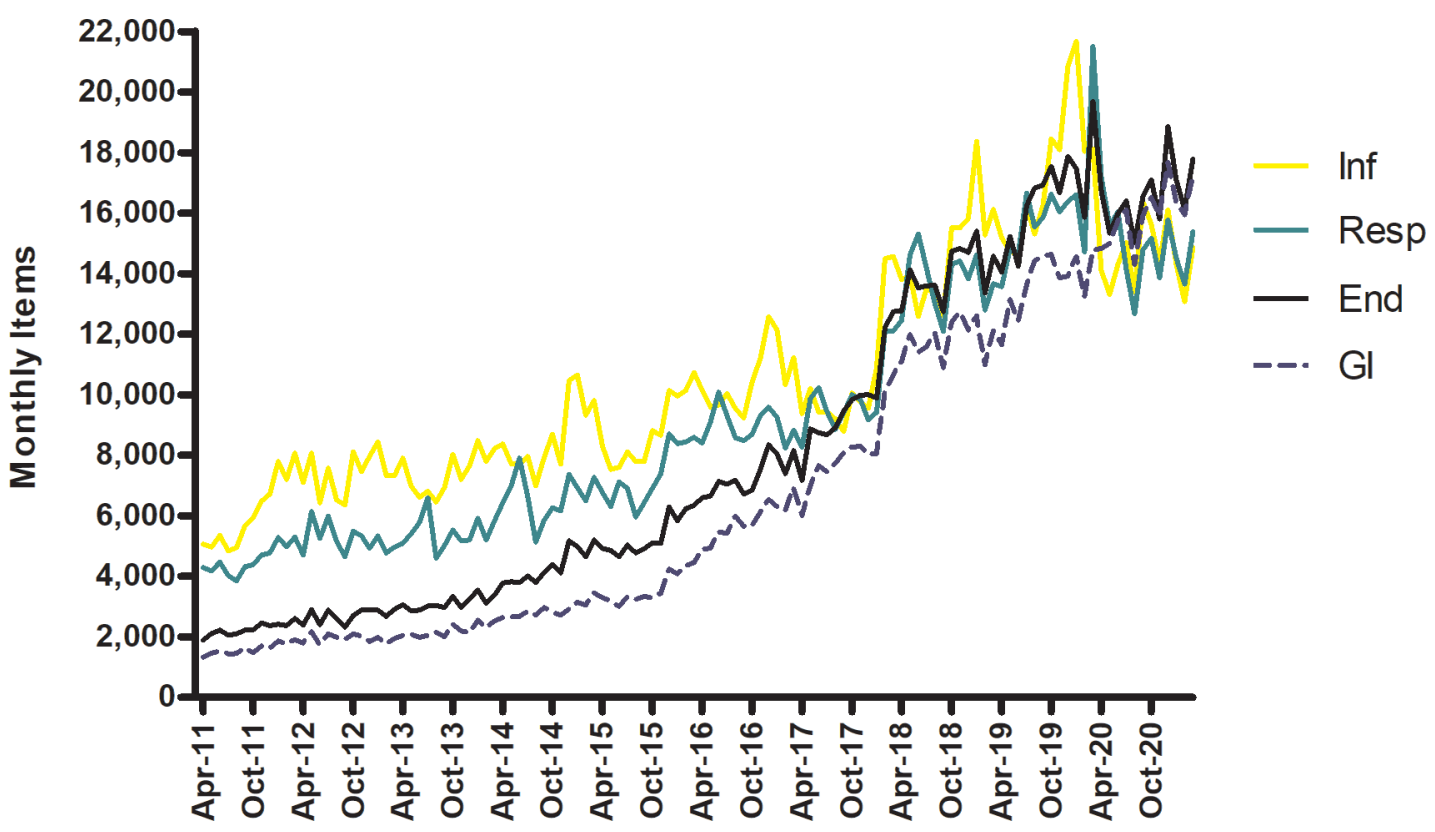

Figure 5 Trend in the number of items prescribed by an NMIP in Wales and dispensed in a community pharmacy by BNF category. The BNF chapters are abbreviated as: End, endocrine system; GI, gastrointestinal system; Inf, infection; Resp, respiratory system.

Vale and Cwm Taf Morgannwg, respectively. The second largest percentage was contributed by respiratory (in three LHBs), cardiovascular (in three LHBs) and nervous system (in one LHB). In 2020-2021, cardiovascular system contributed the largest percentage to the total prescribing in six LHBs, and nervous system contributed the largest percentage in the remaining LHB (Swansea Bay). The second largest percentage in six LHBs was contributed by nervous system, while cardiovascular contributed the second largest percentage in one LHB
(Swansea Bay). This pattern was consistent with data prior to the COVID-19 pandemic in 2018-2019 and 2020-2021.

\section{DISCUSSION}

The total number of items prescribed each year by NMIPs in Wales and dispensed in community pharmacy increased over the study period. In the financial year 2020-2021, total NMIP prescribing was $430 \%$ greater than in $2011-$ 2012, with the largest annual increases seen after April

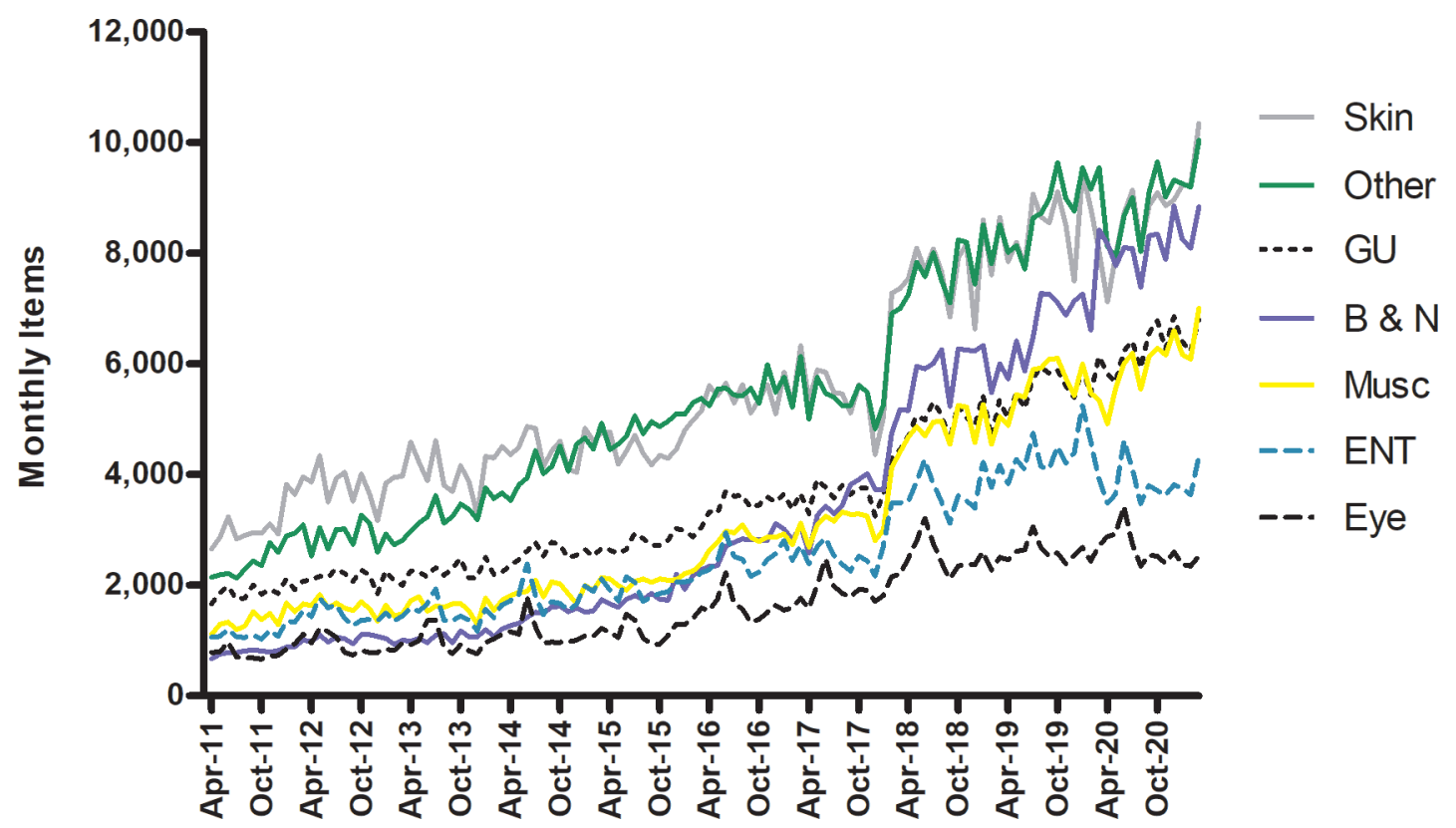

Figure 6 Trend in the number of items prescribed by an NMIP in Wales and dispensed in a community pharmacy by BNF category. The BNF chapters are abbreviated as: B \& N, blood and nutrition; ENT, ears, nose and throat; GU, genito-urinary system; Musc, musculoskeletal system; . 
Table 1 Total prescribing by NMIPs during the whole study period, and in 2011-2012, 2019-2020 and 2020-2021 according to LHB

\begin{tabular}{lccccc}
\hline & Total $\mathbf{n}(\%)$ & 2011-2012n (\%) & 2019-2020 $\mathbf{~ ( \% ) ~}$ & 2020-2021 n (\%) & 2020 mid-year population n (\%) \\
\hline Wales & $11221122(100)$ & $417348(100)$ & $2103637(100)$ & $2214211(100)$ & $3169586(100)$ \\
AB & $2080391(19)$ & $57985(14)$ & $406461(19)$ & $492472(22)$ & $598194(19)$ \\
BC & $3633565(32)$ & $123253(30)$ & $663733(32)$ & $675107(30)$ & $703361(22)$ \\
CV & $1431904(13)$ & $96398(23)$ & $232040(11)$ & $337484(15)$ & $504497(16)$ \\
CTM $^{*}$ & $776162(7)$ & $36229(9)$ & $146266(7)$ & $134557(6)$ & $449836(14)$ \\
HD & $1634835(15)$ & $53232(13)$ & $393483(19)$ & $254026(11)$ & $389719(12)$ \\
Pow & $663344(6)$ & $4405(1)$ & $134943(6)$ & $168304(8)$ & $133030(4)$ \\
SB $^{*}$ & $1000921(9)$ & $45846(11)$ & $126711(6)$ & $152261(7)$ & $390949(12)$ \\
\hline
\end{tabular}

Items prescribed in 2020-2021 correlated with mid-year health board population estimates $\left(r^{2}=0.66, p<0.05\right.$, Pearson correlation analysis).

* The structure of these health boards changed in April 2019 as noted in the methods section

AB, Aneurin Bevan University Health Board; BC, Betsi Cadwaladr University Health Board; CTM, Cwn Taf Morgannwg University Health

Board; CV, Cardiff and Vale University Health Board; HD, Hywel Dda University Health Board; LHBs, local health boards; NMIPs, non-medical independent prescribers; Pow, Powys Teaching Health Board; SB, Swansea Bay University Health Board.

2016. The study identified that over the whole period from April 2011 to March 2021 prescribing in seven BNF chapters equated to approximately $80 \%$ of total items prescribed by NMIPs in Wales, with cardiovascular system the most prescribed chapter. From 2011-2012 to 20192020 , there was a year-on-year increase in prescribed items across the majority of BNF chapters, with the exception of vaccines, (and from 2016- 2017 to 2017-2018 infection). From 2019-2020 to 2020-2021, prescribing fell in five chapters, but with the exception of vaccines, remained higher than in 2011-2012. In the financial year 20112012, the BNF chapters with the greatest items prescribed were infection and respiratory system, while in 2020 2021, the BNF chapters with the greatest items prescribed were cardiovascular and nervous system. The number of items prescribed in each LHB in Wales varied, however, the BNF chapters contributing the largest percentages of items to the LHB totals were broadly comparable.

Prior to 2015-2016, infection was the BNF chapter with the greatest number of items prescribed. A study of nurse prescribing in England with data from 2006 to 2010, similarly identified penicillins as the most prescribed drug category by this professional group..$^{20}$ A year-on-year increase in prescribing of anti-infectives was observed across most years in the current study, consistent with an earlier study of antibiotic prescribing trends in England. ${ }^{21}$ However, over the whole 10-year period of this study, the greatest prescribing was seen in the cardiovascular and nervous system chapters. This was mainly due to large increases in prescribing in these chapters after 20152016. The volume of prescribing indicates that these were the predominant therapeutic areas in which NMIPs were using their qualification after 2015-2016, and indicates a shift in the focus of prescribing compared with the earlier years included in the study. The smaller observed increase in anti-infective prescribing (compared with cardiovascular and nervous system) over the study period may also reflect the role of NMIPs in promoting antimicrobial stewardship and the appropriate prescribing of these medicines. $^{22} 23$

The observed increase in prescribing volume in cardiovascular and nervous system chapters, and the smaller increase in anti-infective prescribing coincided with the introduction of primary care clusters in Wales. This change to the organisational structure of healthcare delivery in Wales has been a driver for the creation of additional pharmacist and advanced nurse practitioner posts within general practices. Funding has been made available in Wales to provide prescribing training for these healthcare professionals, ${ }^{13}$ and the number of NMIPs working in primary care increased over the period from 2011 to $2018 .{ }^{15}$ In this context, the role that pharmacists have in the management of patients with complex, chronic conditions, has been noted. ${ }^{12}$ The increase in the volume of prescribing from the cardiovascular chapter in particular, (along with somewhat smaller increases in prescribing from other chapters associated with of longterm illness such as the endocrine chapter), suggests that a move towards chronic disease management has been realised in practice. The increase in prescribing from the nervous system chapter adds some further support to this. However, in addition to containing medicines for the management of chronic mental illness and neurological disorders, the nervous system chapter also contains medicines for analgesia, which might have been prescribed for acute pain and minor illness (eg, paracetamol for fever). The proportion of non-analgesic prescribing as a percentage of total nervous system chapter items increased from $50 \%$ to $66 \%$ during the study period, providing further evidence in support of an increase in primary care NMIP prescribing for long-term psychological and neurological conditions. An additional complication is the classification of certain medicines within the nervous system chapter. Amitriptyline and the gabapentinoids (gabapentin and pregabalin) are used in the management of depression and epilepsy, respectively, and some 


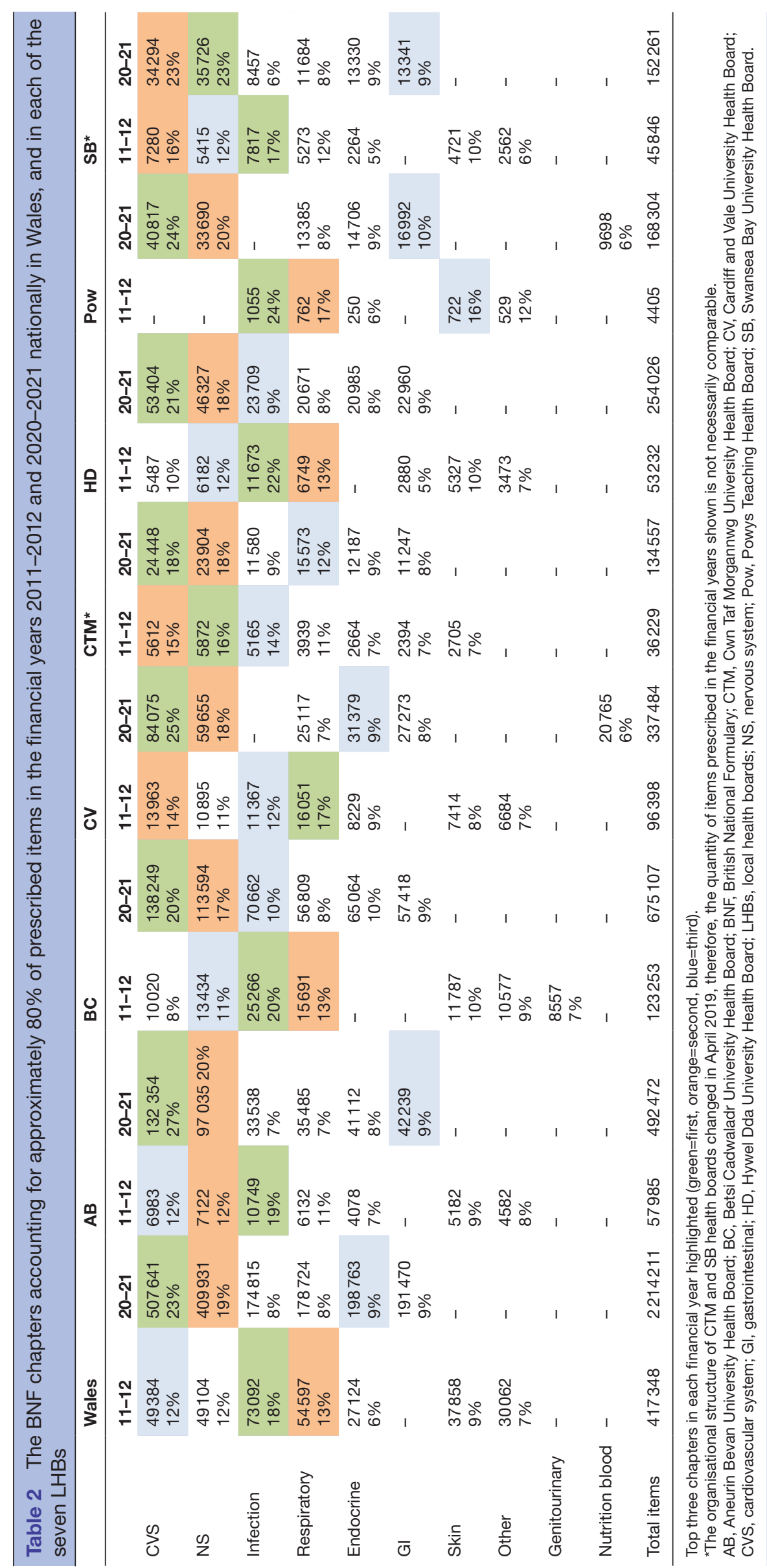


of this prescribing is recorded in those sections of CASPA. However, these medicines may also be prescribed for the management of neuropathic pain, ${ }^{24}$ and some formulations of each medicine are recorded in the analgesia section of CASPA, despite the indication not necessarily being specific to the formulation. Adjusting the categorisation of these medicines to analgesics from 2015 to 2016 onwards (when the greatest increases in prescribing were seen) results in prescribing for long-term (non-analgesic) psychological and neurological conditions accounting for $50 \%$ of items in 2015-2016, increasing to $57 \%$ in 2020 2021. While lower than the unadjusted percentages, there still appears to be an increase in prescribing for these long-term conditions. Consistent with the findings of our study, a survey of pharmacy professionals working in UK General Practice conducted in 2018/2019, identified that over $80 \%$ of pharmacist respondents provided clinical services including chronic disease management. ${ }^{25}$ It should be noted that the sample size was relatively small, with 81 pharmacists, of whom, approximately $75 \%$ were prescribers. A change in prescribing from items for acute to chronic illness was also observed in a study of nurse practitioners prescribing in Ontario (Canada) from 2000 to $2010 .{ }^{26}$ In contrast, a study examining more recent non-medical prescribing trends in New Zealand identified antibiotics and analgesics as the most commonly prescribed items. ${ }^{27}$ However, it should be noted that this study included practitioners able to prescribe from a limited formulary, in addition to NMIPs, which may have influenced the findings. These changes suggest that those responsible for delivering NMIP education should ensure that chronic disease management forms part of the programme of learning.

Over the whole study period, the total number of items prescribed by NMIPs in each of the seven LHBs in Wales varied significantly, with the lowest prescribing in Powys, and the highest in Betsi Cadwaladr. Although the number of items varied, the chapters contributing the greatest number of prescribed items were relatively consistent across the LHBs. Infection accounted for the most items in five of the seven LHBs in 2011-2012, while cardiovascular and nervous system were the two most prescribed chapters across all seven LHBs in 2020-2021. There was a relatively weak correlation between items prescribed and population, which reflected the previous observation that LHBs with larger populations employed greater numbers of NMIPs. ${ }^{15}$ The COVID-19 pandemic appeared to have a variable impact across the LHBs based on the difference in NMIP prescribing between 2019-2020 and 2020-2021. Overall, NMIP prescribing was 5\% higher in Wales in 2020-2021 compared with the previous year, although this was a much smaller increase than the $16 \%$ seen from 2018-2019 to 2019-2020. Across Wales as a whole, NMIP prescribing from respiratory, infection, ear, nose and throat, vaccines, and anaesthesia chapters was lower in 2020-2021 than in the previous year. In the individual LHBs, prescribing in two (Hywel Dda and Cwm Taf Morgannwg) was lower, prescribing in one (Betsi
Cadwaladr) remained relatively constant, and prescribing in the other four LHBs was higher than the previous year. Prescribing of antibiotics decreased in LHBs compared with 2019-2020, consistent with other studies examining primary care usage of these drugs during the COVID-19 pandemic. $^{28} 29$ This COVID-19-related effect contributed to infection being the sixth ranked BNF chapter in 2020-2021, compared with the third ranked chapter in 2019-2020. However, the percentage contribution of the infection chapter to total NMIP prescribing was similar in both years (8\% and $10 \%$, respectively). Other chapters in which there was a reduction in items prescribed across all LHBs were vaccines, and ear, nose and throat. Nevertheless, the data indicate that NMIPs were still actively prescribing as part of their roles during the pandemic.

This study has some limitations. As a result of the retrospective secondary data design, confounding factors that may have influenced prescribing trends could not be identified and explored. ${ }^{30}$ For example, while the introduction of primary care clusters may have been a driver for the apparent change towards more chronic disease management in Wales (as well as the overall increase in prescribing), a causal association could not be determined. CASPA does not provide patient level data, nor data relating to indication, which limits conclusions that can be drawn. While the increase in prescribing from the cardiovascular chapter suggested an increase in chronic disease management, it was not possible to determine whether NMIPs were prescribing ongoing items for the same patient on a long-term basis, or whether they were prescribing occasional items for multiple patients. Similarly, as noted above, indications for the gabapentinoids and amitriptyline could not be determined. The prescribing measure chosen for this study was items, as it was felt that this best reflects a single prescribing interaction. However, it should be noted that the quantity supplied on each prescription may vary. If a prescription were issued with a 3-month supply, the number of items would be smaller. In particular, this may have influenced the number of oral contraceptive items prescribed as part of the genitourinary chapter, as these are typically provided as a longer supply.

In summary, the BNF chapter with the most prescribed items changed from infection to cardiovascular during the study period. This, along with increases in prescribing from other chapters associated with long-term illness, suggested an increase in chronic disease management by NMIPs over time. There was little regional variation in the therapeutic areas most commonly prescribed, indicating a similar approach to NMIP implementation across the LHBs in Wales. The COVID-19 pandemic had variable impact on prescribing both in terms of therapeutic area, and regional variation. However, prescribing by NMIPs continued to increase, and the impact of this on the delivery of primary care services is a focus for future work.

Twitter Karen Hodson @HodsonKL 
Contributors PD and HB made a substantial contribution to the conception and design of the work; the acquisition, analysis and interpretation of data; and drafting ofthe work. $\mathrm{KHa}, \mathrm{KHo}$ and $\mathrm{RD}$ made a substantial contribution to the design of the work, acquisition, analysis and interpretation of data. All authors criticallyrevised drafts of the work and approved the final version to be published and agree to be accountable for all aspects of the work in ensuring that questions related to the accuracy or integrity of any part of the work are appropriately investigated and resolved. $\mathrm{PD}$ is the author acting as guarantor.

Funding The authors have not declared a specific grant for this research from any funding agency in the public, commercial or not-for-profit sectors.

Competing interests None declared.

Patient and public involvement Patients and/or the public were not involved in the design, or conduct, or reporting, or dissemination plans of this research.

Patient consent for publication Not applicable.

Ethics approval The database (CASPA) used in this study holds routinely collected, anonymised prescription data, and did not allow the researcher to access any patient or prescriber identifiable information. As such ethical approval was not required for this study. This study was part of a wider project, which was approved by the researcher's LHB research and development department (reference number: 14/CLC/5882).

Provenance and peer review Not commissioned; externally peer reviewed. Data availability statement № data are available.

Open access This is an open access article distributed in accordance with the Creative Commons Attribution Non Commercial (CC BY-NC 4.0) license, which permits others to distribute, remix, adapt, build upon this work non-commercially, and license their derivative works on different terms, provided the original work is properly cited, appropriate credit is given, any changes made indicated, and the use is non-commercial. See: http://creativecommons.org/licenses/by-nc/4.0/.

ORCID iD

Paul Deslandes http://orcid.org/0000-0001-6751-9438

\section{REFERENCES}

1 Cope LC, Abuzour AS, Tully MP. Nonmedical prescribing: where are we now? Ther Adv Drug Saf 2016;7:165-72.

2 Graham-Clarke E, Rushton A, Noblet T, et al. Non-Medical prescribing in the United Kingdom National health service: a systematic policy review. PLoS One 2019;14:e0214630.

3 Department of Health. Review of prescribing, supply \& administration of medicines. Final report, 1999. Available: https://www.publichealth. hscni.net/sites/default/files/directorates/files/Review\%20of\% 20prescribing,\%20supply\%20and\%20administration\%20of\% 20medicines.pdf [Accessed 04 Jan 2022].

4 Department of Health. The NHS plan: a plan for investment. A plan for reform, 2000. Available: https://webarchive.nationalarchives. gov.uk/20130124064356/http://www.dh.gov.uk/prod_consum_dh/ groups/dh_digitalassets/@dh/@en/@ps/documents/digitalasset/dh_ 118522.pdf [Accessed 16 Oct 2020].

5 Stewart DC, Maclure K, Bond CM, et al. Pharmacist prescribing in primary care: the views of patients across great Britain who had experienced the service. Int J Pharm Pract 2011;19:328-32.

6 Deslandes R, John DN, Deslandes PN. An exploratory study of the patient experience of pharmacist supplementary prescribing in a secondary care mental health setting. Pharm Pract 2015;13:553.

7 Tinelli M, Blenkinsopp A, Latter S, et al. Survey of patients' experiences and perceptions of care provided by nurse and pharmacist independent prescribers in primary care. Health Expect 2015; 18:1241-55.

8 Mills T, Patel N, Ryan K. Pharmacist non-medical prescribing in primary care. A systematic review of views, opinions, and attitudes. Int J Clin Pract 2021;75:e13827.

9 Hindi AMK, Seston EM, Bell D, et al. Independent prescribing in primary care: a survey of patients', prescribers' and colleagues' perceptions and experiences. Health Soc Care Community 2019;27:e459-70.

10 Courtenay M, Khanfer R, Harries-Huntly G, et al. Overview of the uptake and implementation of non-medical prescribing in Wales: a national survey. BMJ Open 2017a;7:e015313.

11 Stewart D, Jebara T, Cunningham S, et al. Future perspectives on nonmedical prescribing. Ther Adv Drug Saf 2017;8:183-97.

12 National Assembly for Wales, Health, Social Care and Sport Committee. Inquiry into primary care: clusters, 2017. Available: https://senedd.wales/laid\%20documents/cr-ld11226/cr-ld11226-e. pdf

13 Welsh Government. Our plan for a primary care service for Wales up to March 2018, 2014. Available: http://www.wales.nhs.uk/sitesplus/ documents/986/our\%20plan\%20for\%20primary\%20care\%20in\% 20wales\%20up\%20to\%20march\%202018.pdf [Accessed 20 Nov 2020].

14 Welsh Government. A healthier Wales: our plan for health and social care, 2018. Available: https://gov.wales/sites/default/files/ publications/2019-10/a-healthier-wales-action-plan.pdf

15 Alghamdi SSA, Hodson K, Deslandes P, et al. Prescribing trends over time by non-medical independent prescribers in primary care settings across Wales (2011-2018): a secondary database analysis. BMJ Open 2020;10:e036379.

16 All Wales Therapeutics and Toxicology Centre. National prescribing indicators 2020-2021 analysis of prescribing data to March 2021 2021. Available: https://awmsg.nhs.wales/files/national-prescribingindicators/national-prescribing-indicators-2020-2021-analysis-ofprescribing-data-to-march-2021-pdf/

17 Groves S, Cohen D, Alam MF, et al. Abolition of prescription charges in Wales: the impact on medicines use in those who used to pay. Int $J$ Pharm Pract 2010;18:332-40.

18 Welsh Government. Population estimates by local health boards and year, 2020. Available: https://statswales.gov.wales/Catalogue/ Population-and-Migration/Population/Estimates/Local-HealthBoards/populationestimates-by-welshhealthboard-year

19 Joint Formulary Committee. British National formulary. BMJ group and pharmaceutical press, 2021.

20 Drennan VM, Grant RL, Harris R. Trends over time in prescribing by English primary care nurses: a secondary analysis of a national prescription database. BMC Health Serv Res 2014;14:54.

21 Courtenay M, Gillespie D, Lim R. Patterns of dispensed nonmedical prescriber prescriptions for antibiotics in primary care across England: a retrospective analysis. $J$ Antimicrob Chemother 2017b;72:2915-20.

22 Courtenay M, Rowbotham S, Lim R, et al. Antibiotics for acute respiratory tract infections: a mixed-methods study of patient experiences of non-medical prescriber management. BMJ Open 2017c;7:e013515.

23 Hodson K, Deslandes R, Courtenay M. Pharmacist independent prescribers can make a significant contribution to antimicrobial stewardship. Pharm. J 2017;298.

24 National Institute for Health and Care Excellence. Neuropathic pain in adults: pharmacological management in non-specialist settings CG173, 2013. Available: https://www.nice.org.uk/guidance/cg173

25 Savickas V, Foreman E, Ladva A, et al. Pharmacy services and role development in UK general practice: a cross-sectional survey. Int $J$ Pharm Pract 2021;29:37-44.

26 Tranmer JE, Colley L, Edge DS, et al. Trends in nurse practitioners' prescribing to older adults in Ontario, 2000-2010: a retrospective cohort study. CMAJ Open 2015;3:E299-304.

27 Raghunandan R, Marra CA, Tordoff $\mathrm{J}$, et al. Examining non-medical prescribing trends in New Zealand: 2016-2020. BMC Health Serv Res 2021;21:418.

28 Hussain AZ, Paudyal V, Hadi MA. Impact of the COVID-19 pandemic on the prescribing patterns of first-line antibiotics in English primary care: a longitudinal analysis of national prescribing dataset. Antibiotics 2021;10:591.

29 Zhu N, Aylin P, Rawson T, et al. Investigating the impact of COVID-19 on primary care antibiotic prescribing in North West London across two epidemic waves. Clin Microbiol Infect 2021;27:762-8.

30 Cheng HG, Phillips MR. Secondary analysis of existing data: opportunities and implementation. Shanghai Arch Psychiatry 2014;26:371-5. 